\title{
The bistability of higher-order differences of discrete periodic signals
}

\author{
AY Shahverdian ${ }^{1 *}$, RP Agarwal ${ }^{2}$ and RB Benosman ${ }^{3}$
}

\footnotetext{
"Correspondence: svrdn@yerphi.am ${ }^{1}$ Institute for Informatics and Automation Problems, National Academy of Sciences of Armenia, Paruir Sevak St. 1, Yerevan, 0014, Armenia

Full list of author information is available at the end of the article
}

\begin{abstract}
A theorem on higher-order absolute differences taken from successive terms of bounded sequences is proved. This theorem establishes the property of bistability of such difference series and suggests a method for converting periodic discrete-time signals into the binary digital form based only on the computation of absolute differences.
\end{abstract}

MSC: $37 \mathrm{E} 05 ; 37 \mathrm{M} 10 ; 94 \mathrm{~A} 12$

Keywords: higher-order differences; discrete dynamical systems; discrete-time signals

\section{Introduction}

This paper advances an approach in dynamical systems [1-5] which is based on considering higher-order differences taken from the orbits of a given system. Such an approach is motivated by the observation that some natural systems process the information contained in the signals' difference structure. For example, it is claimed in brain theory that the visual cortex 'responds to contrast rather than the uniform luminosity' and the higher differences up to fourth order in these problems are considered [6].

The difference method reveals some new aspects in analyzing arbitrary time series and discrete dynamical systems. For instance, the difference characteristic $\gamma$ introduced in [2, 3] demonstrates a strong correlation with the Lyapunov exponent [7] used in deterministic chaos (the definition of $\gamma$ can be found in $[2,3]$; see $[8,9]$ for its modification and discussions). Another possible generalization could relate to fractional analysis: it would be interesting to define the fractional analog of $\gamma$ and apply it to chaos discrimination in fractional dynamical systems (such systems are studied, e.g., in [10-12]).

In the present paper another new aspect of the difference analysis, the bistability of higher-order differences taken from a bounded time series (discrete-time signals) is proved. Some possible applications to digital communication and signal processing in Section 2 are considered.

The content of this paper is the following. In Section 2 our main Theorem 1 is formulated. This theorem establishes the property of bistability for higher-order absolute differences taken from discrete-time signals. In the sense of applications it asserts that some systems operating on the basis of their inputs' higher-order differences should rather be classified over digital ones. The theorem suggests a method for converting some discrete (and sampled analog) signals into a binary digital form based only on computation of absolute differences; if applicable (e.g., as for the case of arbitrary periodic signals), this method 
is more effective than the traditional ones used in communication theory. The proof of Theorem 1, based on a version of notion of peak sets originated in Banach algebras and approximation theory, is presented in Section 3.

\section{Main results}

In the difference analysis a given time series (or an orbit of a dynamical system) $X$ is decomposed into two constituents-the sign and magnitude components. The sign component $\mathrm{S}$ reflects the alternation in monotony (increase/decrease) of higher-order absolute differences taken from successive terms of $X$ and does not depend on their exact values. The magnitude (or height) component $\mathrm{H}$ consists of these absolute differences and does not depend on the sign distribution.

Let us proceed to formal definitions. Let

$$
X=\left(f_{1}, f_{2}, \ldots, f_{n}, \ldots\right)
$$

be an infinite bounded numerical sequence-this can be some time series, discrete-time signal, experimental data (long enough), or an orbit of iterates of some map. For natural $m$ we consider $m$ th-order absolute differences

$$
H_{n}^{(0)}=f_{n}, \quad H_{n}^{(m+1)}=\left|H_{n+1}^{(m)}-H_{n}^{(m)}\right| \quad(m \geq 0, n \geq 1)
$$

and define the $m$ th absolute difference sequence as $H_{m}=\left(H_{n}^{(m)}\right)_{n=1}^{\infty}$. Furthermore, we define

$$
S_{m}=\left(\delta_{1}^{(m)}, \delta_{2}^{(m)}, \ldots, \delta_{n}^{(m)}, \ldots\right), \quad \text { where } \delta_{n}^{(m)}= \begin{cases}+1, & H_{n+1}^{(m)} \geq H_{n}^{(m)}, \\ -1, & H_{n+1}^{(m)}<H_{n}^{(m)}\end{cases}
$$

The matrices $\mathrm{S}=\left\{\delta_{n}^{(m)}: m \geq 0, n \geq 1\right\}$ and $\mathrm{H}=\left\{H_{n}^{(m)}: m \geq 0, n \geq 1\right\}$ are called S- and Hcomponents of $X$, the sequences $S_{m}$ and $H_{m}$ are called $m$ th S- and H-components of $X$ and denoted $S_{m}=S_{m}(X)$ and $H_{m}=H_{m}(X)$. In what follows instead of $H_{n}^{(m)}$ we use the notation $H_{n}^{m}$ (omitted brackets).

The next proposition (see also [5]) states that $H_{n}^{m}$ can be written as some linear forms of terms of $X$. This is a consequence of Eq. (2) and the proof, which can be conducted by the induction method, is straightforward (omitted). The $\mathbb{N}, \mathbb{Z}$, and $\mathbb{R}$ denote the collections of natural, integer, and real numbers, respectively; $\left(\begin{array}{c}m \\ i\end{array}\right)\left(=\frac{m !}{i !(m-i) !}\right)$ denotes the binomial coefficients.

Proposition 1 Let $X=\left(f_{n}\right)_{n=1}^{\infty}$ and $m \geq 1$ be arbitrary. Then the $m$ th-order absolute differences $H_{n}^{m}$ can be represented as

$$
H_{n}^{m}=k_{m, n}^{(0)} f_{n}+k_{m, n}^{(1)} f_{n+1}+\cdots+k_{m, n}^{(m)} f_{n+m}
$$

where the coefficients $k_{m, n}^{(i)} \in \mathbb{Z}$ are constructed recurrently: for $p \geq 1$

$$
k_{1, n}^{(0)}=-k_{1, n}^{(1)}=-\delta_{n}^{(0)}, \quad k_{p+1, n}^{(i)}= \begin{cases}-\delta_{n}^{(p)} k_{p, n}^{(i)}, & i=0, \\ \delta_{n}^{(p)}\left(k_{p, n+1}^{(i-1)}-k_{p, n}^{(i)}\right), & 1 \leq i \leq p, \\ \delta_{n}^{(p)} k_{p, n+1}^{(i-1)}, & i=p+1 .\end{cases}
$$


One can see that $k_{m, n}^{(i)}$ in Eq. (4) depend only on S-components $S_{1}, \ldots, S_{m}$ of $X$. The rule (5) is a generalization of the additive scheme for constructing the Pascal triangle of binomial coefficients. It can be proved (we omit this) that for $m, n \geq 1$

$$
\sum_{i=0}^{m} k_{m, n}^{(i)}=0 \quad \text { and } \quad\left|k_{m, n}^{(i)}\right| \leq\left(\begin{array}{c}
m \\
i
\end{array}\right)
$$

The equality sign here is assumed when $\delta_{n}^{(m)}=(-1)^{m}$ for all $n \geq 1$; for this case, (4) gains the form

$$
H_{n}^{m}=\sum_{i=0}^{m}(-1)^{i}\left(\begin{array}{c}
m \\
i
\end{array}\right) f_{n+i}
$$

which is the so-called $\delta$-transform (of the sequence $X$ ) studied in the Hausdorff transforms of divergent series [13]. This particular sign distribution appears also in definition of completely monotonic functions (see, e.g., the Bernstein theorem in [14]).

In this paper we are interested in the $\mathrm{H}$-components of bounded sequences (discretetime signals). To formulate our main theorem, Theorem 1, we present Definition 1 - namely in the context of this definition the (physics-related) term "bistability" in Section 1 is understood. In what follows, for bounded $X=\left(x_{i}\right)_{i=1}^{\infty}$ we consider the sup-norm:

$$
\|X\|=\sup \left\{\left|x_{i}\right|: 1 \leq i<\infty\right\}
$$

A finite subsequence $h=\left(x_{i+1}, \ldots, x_{i+k}\right)$ of $X=\left(x_{i}\right)_{i=1}^{\infty}$ is called a segment of $X$ of length $k$, $|h|=k$; for two functions of natural argument $a(m)$ and $b(m)$, differed from 0 we denote $a(m) \sim b(m)$ if $a(m) / b(m) \rightarrow 1$ as $m \rightarrow \infty$. For a number $x \in \mathbb{R}$ we denote $[x]$ and $\{x\}$ its entire and fractional parts, respectively.

Definition 1 Let the numbers $0 \leq \mu<1$ and $0 \leq \varepsilon<1$ be given. A sequence $X=\left(x_{i}\right)_{i=1}^{\infty}$ is called $\mu$-binary if $x_{i} \in\{0, \mu\}$ for every $i \geq 1 ; X$ is called $(\mu, \varepsilon)$-binary if there exists $\mu$ binary $Z=\left(z_{i}\right)_{i=1}^{\infty}$ such that $\|X-Z\| \leq \varepsilon$. A collection of sequences $\left(X_{m}\right)_{m \geq 1}$ is called $\mu$ binary $\left((\mu, \varepsilon)\right.$-binary) if $X_{m}$ is $\mu$-binary $\left((\mu, \varepsilon)\right.$-binary) for all large enough $m$; $\left(X_{m}\right)_{m \geq 1}$ is called asymptotically $\mu$-binary if for arbitrary $\varepsilon>0$ it is $(\mu, \varepsilon)$-binary.

The $(\mu, 0)$-binary sequences are $\mu$-binary sequences, $(0, \varepsilon)$-binary sequences are the sequences upper bounded by $\varepsilon$. For the case of finite sequences $X$ of length $n$ the above-given definitions are analogous, replacing in Eq. (7) and in Definition 1 the symbol $\infty$ by $n+1$ and $n$, respectively.

The proof of next proposition is straightforward (and is omitted).

Proposition 2 A collection of sequences $\left(X_{m}\right)_{m \geq 1}$ is asymptotically $\mu$-binary if and only if there exists a $\mu$-binary collection $\left(Z_{m}\right)_{m \geq 1}$ such that $\left\|X_{m}-Z_{m}\right\| \rightarrow 0$ as $m \rightarrow \infty$.

Theorem 1 is the main result of this paper. We define some notions involved in its formulation. For $X=\left(f_{n}\right)_{n=1}^{\infty}$ and $H_{m}=H_{m}(X)$ we consider

$$
\mu_{m}=\left\|H_{m}\right\| \quad \text { and } \quad \mu=\lim _{m \rightarrow \infty} \mu_{m} \quad(\mu \geq 0) .
$$


For periodic $X$ the supremum in Eq. (7) can be replaced by maximum. We note that Eq. (2) yields the result that for every $X$ we have $\mu_{m+1} \leq \mu_{m}(m \geq 1)$. Due to this monotony of $\mu_{m}$, for arbitrary $X$ one and only one of next two options (a) and (b) is possible:
(a) $\mu=\mu_{m}$ for all large enough $m$,
(b) $\quad \mu<\mu_{m}$ for $m=1,2, \ldots$

We define the peak sets $P_{m}$,

$$
P_{m}=\left\{n \geq 1: H_{n}^{m} \geq \mu\right\}
$$

(it follows from Eq. (8) that $P_{m} \neq \emptyset$ for $m \geq 1$ ), modifying the corresponding notion from theory of Banach algebras (e.g., [14]). Let $P_{m}$ be numbered in increasing order of its entries,

$$
P_{m}=\left\{n_{m}^{(1)}, n_{m}^{(2)}, \ldots\right\} \quad \text { and denote } \quad d_{m}=\max \left\{\left|n_{m}^{(i+1)}-n_{m}^{(i)}\right|: i \geq 1\right\}
$$

In item (b) of Theorem 1 the following restrictions on $P_{m}$ are imposed:

$$
1 \leq d_{m} \leq m, \quad \lim _{m \rightarrow \infty}\left(m-d_{m}\right)=\infty
$$

The $d_{m}$ characterize the density of $P_{m}$ in natural series $\mathbb{N}$. The first condition in (12) means that $P_{m}$ is dense enough in $\mathbb{N}$ (every segment of $\mathbb{N}$ of length $m$ contains a point of $P_{m}$ ); the second relation implies that the set

$$
E=\left\{m-d_{m}: m \in \mathbb{N}\right\}, \quad E \subseteq \mathbb{N}
$$

is infinite. If $n \in E$, then there is some $m \geq 1$ such $n=m-d_{m}$; we denote such an $m$ as $m=m_{n}$; hence, one can consider a function of the natural argument,

$$
\varphi: \mathbb{N} \rightarrow \mathbb{N}, \quad \text { defined as } \quad \varphi(n)=m_{n}-d_{m_{n}} .
$$

For example, if $d_{m}=[m / 2]$ (entire part of half of $m$ ), then (12) is satisfied and one can assign $\varphi(n)=[n / 2]$; another example is

$$
\left.d_{m}=T \text { (= const. }\right), \quad \text { and then } \quad \varphi(n)=n-T .
$$

In these examples, $E$ coincides with $\mathbb{N}$; Eq. (15) holds, e.g., for $T$-periodic $X$.

The next theorem establishes the bistability of higher-order absolute differences taken from discrete signals. In addition, it asserts (descriptively) that the denser the peak set $P_{m}$ is, the denser is the asymptotically binary $H_{m}$.

Theorem 1 Let $X=\left(f_{n}\right)_{n=1}^{\infty}, H_{m}=H_{m}(X)$, and $\mu_{m}$ and $\mu$ be defined by Eq. (8). Then the following statements, provided that a finite collection (whose cardinality is smaller than $\left.d_{m}\right)$ of entries of $H_{m}$ can be excluded, are true:

(a) For every $m \in \mathbb{N}$ there exists a finite segment $h_{m} \subset H_{m}$ such that $\left|h_{m}\right| \sim m$ as $m \rightarrow \infty$ and the collection $\left(h_{m}\right)_{m=1}^{\infty}$ is asymptotically $\mu$-binary. 
(b) If the peak sets (10) satisfy Eq. (12) then the collection $\left(H_{n}\right)_{n \in E}$, where $E$ is defined by Eq. (13), is asymptotically $\mu$-binary; more precisely, for every $n \in E$ the $H_{n}$ is $\left(\mu, \varepsilon_{n}\right)$-binary with $\varepsilon_{n}=\mu_{\varphi(n)}-\mu$ where $\varphi$ is defined by Eq. (14).

(c) Let $X$ be periodic with a period $T \geq 1$. Then the collection $\left(H_{m}\right)_{m=1}^{\infty}$ is either $\mu$-binary or asymptotically $\mu$-binary. More precisely, if Eq. (9a) holds, $\left(H_{m}\right)_{m=1}^{\infty}$ is $\mu$-binary, and if Eq. (9b) holds, $\left(H_{m}\right)_{m=1}^{\infty}$ is asymptotically $\mu$-binary: for every $m>T$ the $H_{m}$ is $\left(\mu, \varepsilon_{m}\right)$-binary with $\varepsilon_{m}=\mu_{m-T}-\mu$.

The condition Eq. (12) on peak sets in this theorem is imposed on the difference series $H_{m}$, but not immediately on $X$, which leads to certain difficulties to decide whether for a given $X$ its higher order difference series possess the asymptotic bistability. Theorem 1(c) asserts that this property always holds for arbitrary periodic $X$.

In the next corollary both options from Theorem 1(c) are presented (below, the real numbers $f_{1}, \ldots, f_{T}$ are called H-independent if for arbitrary $\lambda_{1}, \ldots, \lambda_{T} \in \mathbb{Z}$ for which $\lambda_{1}+$ $\cdots+\lambda_{T}=0$, the relation $\sum_{i=1}^{T} \lambda_{i} f_{i}=0$ implies that all the $\lambda_{i}$ are zero):

Corollary 1 Let $X=\left(f_{n}\right)_{n=1}^{\infty}$ be periodic with a period $T \geq 1, H_{m}=H_{m}(X)$, and let $\mu$ be defined by Eq. (8). Then if $f_{1}, \ldots, f_{T}$ are rational numbers then the collection $\left(H_{m}\right)_{m=1}^{\infty}$ is $\mu$ binary, and if $f_{1}, \ldots, f_{T}$ are $\mathrm{H}$-independent then $\left(H_{m}\right)_{m=1}^{\infty}$ is asymptotically $\mu$-binary.

Let us discuss the reason why a bistability, considered in Theorem 1, is emerged. Despite the $X$ (the line $H_{0}$ in the matrix $\mathrm{H}$ ) can be arbitrary, Eq. (2) sets a strong interrelation between the entries of every triplet $\left\{H_{n}^{m+1}, H_{n}^{m}, H_{n+1}^{m}\right\}$ of the matrix $H$. Namely such intrinsic local restrictions are usually the reason of some special features (in our case this is the bistability) of a given object (in our case this is the matrix H); e.g., the maximum principle for harmonic functions (both classical and discrete [15] versions) is due to their local mean value property. For proving Theorem 1 we exploit the following situation: if the absolute difference $\left(=H_{n}^{m+1}\right)$ of two positive quantities $\left(H_{n}^{m}\right.$ and $\left.H_{n+1}^{m}\right)$ is close to their upper bound, then one of them should also be close to this bound while another one should be close to zero. Similar situations arise also in Banach algebras and approximation theory, see, e.g., the Bishop-de Leeuw theorem ([14], Ch. 8), Bishop's “ $\frac{1}{4}-\frac{3}{4}$ ”-criterion ([16], Ch. II, Comments) and Mergelyan's earlier work [17] on rational approximations where a binary-valued function $\mathfrak{m}(\zeta)$ is of the main interest.

Before discussing on applications of Theorem 1 we note that a given signal $X=\left(f_{n}\right)_{n=1}^{\infty}$ can be completely restored by its first entries and components $\mathrm{S}$ and $\mathrm{H}$. Namely, the next proposition (see also [5]; the proof is by the induction method and is omitted) provides us with analytical expressions for the computation of the original $X$ by given $S_{1}, \ldots, S_{m}, H_{m}$ and the first terms $f_{1}, \ldots, f_{m}$ of $X$ (or, by first terms of $H_{1}^{m}$ denoted now as $H_{1}^{k}=\left[f_{1}, \ldots, f_{k}\right]$, $2 \leq k \leq m)$.

Proposition 3 Let $m \geq 1$ be given, let $H=\left(h_{n}\right)_{n=1}^{\infty}$ be some infinite sequence, $h_{n} \geq 0$ and $S_{k}=\left(s_{k, n}\right)_{n=1}^{\infty}, s_{k, n}= \pm 1,0 \leq k \leq m$ be some infinite binary sequences. Let $X=\left(f_{n}\right)_{n=1}^{\infty}$ be defined as follows: $f_{1}, \ldots, f_{m}$ are arbitrary and for every natural $n \geq 1$

$$
f_{n}=f_{1}+\sum_{k=2}^{m}\left[f_{1}, \ldots, f_{k}\right] \sum_{i=1}^{n-k+1} B_{n, i, k-2}+\sum_{i=1}^{n-m} h_{i} B_{n, i, m-1},
$$


where $\sum_{i}^{j}=0$ if $j<i$ and $B_{n, i, p} \in \mathbb{Z}$ are constructed recurrently: for $p \geq 1$

$$
B_{n, i, 0}=s_{0, i}, \quad B_{n, i, p}=s_{p, i} \sum_{j=i+1}^{n-p} B_{n, j, p-1} .
$$

Then for all $1 \leq k \leq m$ the relations

$$
S_{k}(X)=S_{k} \quad \text { and } \quad H_{m}(X)=H
$$

hold.

Let us outline some possible applications of the above-presented theory to signal processing. Theorem 1 suggests a method for converting the discrete signals into the binary ones based only on the computation of differences. For processing the signals $X=\left(f_{n}\right)_{n=1}^{\infty}$ for which $\mu_{m}$ converges to $\mu$ fast enough, this method can be far more effective than the ones which deal with replacing $f_{n}$ by their binary codes. Namely, we claim that, when applicable (e.g., if $X$ is periodic), the difference converting method can reduce the data to be transmitted and can increase significantly the transmission speed. One can suggest the following scheme for processing (not only the transmission) the discrete-time signals by the digital systems: by Theorem 1 (and using Proposition 1) a given $X$ is converted into a binary form, which is then processed as a digital signal, and then the obtained (binary) signal is deconverted (provided that a number of S-components and some finite set of the resulting signal entries are given) by Proposition 3.

To illustrate this, we consider the periodic case. Let a signal $X=\left(f_{n}\right)_{n=1}^{\infty}$ be periodic; by using the binary symbols it should be transmitted (it suffices to transmit the period $f_{1}, \ldots, f_{T}$ ). The traditional method, where each of the $f_{i}$ is replaced by a binary code of some length $q$, requires $q T$ binary digits (bits) to be transmitted. By the difference method, this transmission task is solved as follows. Let $m$ be a minimal number for which the sequence $H_{m}$ can be treated as a binary one (the $\varepsilon_{m}$ from Theorem 1 is small enough). According to Proposition 3, to transmit $X$ one should transmit $m(q+T+2)$ bits $((m+1) q$ of them to transmit $f_{1}, \ldots, f_{m}$ and $\mu$ by usual method, $m T$ to transmit $S_{1}, \ldots, S_{m}$, and $m$ bits to transmit $H_{m}$ ). If $m<T$, the ratio

$$
\frac{m(q+T+2)}{q T}
$$

can be smaller than 1, i.e., the difference method indeed is more effective; e.g., if $f_{i}=i$ $(1 \leq i \leq T)$, then $m=2$ and the above-mentioned ratio is indeed small if $q$ and $T$ are large.

\section{Proof of Theorem 1}

The proof of Theorem 1 is based on the following lemma, where the notion of peak sets defined by Eq. (10) is essentially used.

Lemma 1 Let $X=\left(f_{n}\right)_{n=1}^{\infty}, H_{m}=H_{m}(X), \mu_{m}$ and $\mu$ be defined by Eq. (8), and for some $m, n \geq$ 1 , the inequality

$$
H_{n}^{m} \geq \mu
$$


holds. Then for every $0 \leq k \leq m$ and every $0 \leq i \leq k$ one of the two relations (19) or (20),

$$
\begin{aligned}
& \mu \leq H_{n+i}^{m-k} \leq \mu_{m-k} \quad \text { and } \quad 0 \leq H_{n+i+1}^{m-k} \leq \mu_{m-k}-\mu \text {, } \\
& \mu \leq H_{n+i+1}^{m-k} \leq \mu_{m-k} \quad \text { and } \quad 0 \leq H_{n+i}^{m-k} \leq \mu_{m-k}-\mu \text {, }
\end{aligned}
$$

holds; that means that for every $1 \leq k \leq m$ the finite sequence

$$
H_{n}^{m-k}, H_{n+1}^{m-k}, \ldots, H_{n+k}^{m-k}
$$

is $(\mu, \varepsilon)$-binary with $\varepsilon=\mu_{m-k}-\mu$.

Proof The proof is conducted by the induction method with respect to the variable $k$, $1 \leq k \leq m-1$. Let us first prove the lemma for the case $k=1$. From definition of $\mu_{m}$ and $\mu$, we have

$$
\mu \leq H_{n}^{m} \leq \mu_{m}, \quad \text { that is, by Eq. (2), } \quad \mu \leq\left|H_{n}^{m-1}-H_{n+1}^{m-1}\right| \leq \mu_{m} .
$$

Let us assume that $H_{n}^{m-1} \geq H_{n+1}^{m-1}$ (for the contrary case $H_{n}^{m-1} \leq H_{n+1}^{m-1}$ the proof is analogous); that is, the second relation in Eq. (22) can be written as

$$
\mu \leq H_{n}^{m-1}-H_{n+1}^{m-1} \leq \mu_{m}
$$

From the left-hand side of (23) we obtain $\mu \leq H_{n}^{m-1}$, and from the definition in Eq. (8) it follows that $H_{n}^{m-1} \leq \mu_{m-1}$, that is, $\mu \leq H_{n}^{m-1} \leq \mu_{m-1}$. Furthermore, from Eq. (23) one obtains

$$
H_{n+1}^{m-1} \leq H_{n}^{m-1}-\mu \leq \mu_{m-1}-\mu
$$

and, hence, Lemma 1 for the case $k=1$ is proved.

To prove the lemma for arbitrary $2 \leq k \leq m$, we apply the induction method: we assume that one of the relations (19) or (20) for some $1 \leq k \leq m-2$ holds, and we prove that it holds also when $k$ is substituted by $k+1$. Let us assume that Eq. (19) holds (for the case of Eq. (20) the proof is analogous). By Eq. (2)

$$
H_{n+i}^{m-k}=\left|H_{n+i}^{m-(k+1)}-H_{n+i+1}^{m-(k+1)}\right|
$$

and let us assume that the difference here is positive (if it is negative, the proof is the same),

$$
H_{n+i}^{m-k}=H_{n+i}^{m-(k+1)}-H_{n+i+1}^{m-(k+1)} .
$$

From (19) we have $H_{n+i}^{m-(k+1)} \geq \mu$. We also have

$$
H_{n+i}^{m-(k+1)} \leq \mu_{m-(k+1)}=\mu+\left(\mu_{m-(k+1)}-\mu\right)
$$


and, hence, the first relation in Eq. (19), where $k$ is substituted by $k+1$, is proved. Let us now consider the second relation in (19). Eqs. (24) and (19) give

$$
H_{n+i+1}^{m-(k+1)}=H_{n+i}^{m-(k+1)}-H_{n+i}^{m-k} \leq \mu_{m-(k+1)}-\mu=\varepsilon_{m-(k+1)}
$$

and Eqs. (19) and (20), where $k$ is substituted by $k+1$, are proved. Lemma 1 is proved.

Proof of Theorem 1 (a) Let $X=\left(f_{n}\right)_{n=1}^{\infty}$ be given, $H_{m}=H_{m}(X)$, and $\mu_{m}$ and $\mu$ be defined by Eq. (8). It follows from definition of $\mu$ that for every $m \geq 1$ there is some $n=n_{m}$ such that $H_{n}^{m} \geq \mu$ (cf. Eq. (18)). Then by Lemma 1 for every $1 \leq k \leq m$ the sequence

$$
H_{n}^{m-k}, H_{n+1}^{m-k}, \ldots, H_{n+k}^{m-k}
$$

is $\left(\mu, \mu_{m-k}-\mu\right)$-binary. Let $k(m)$ be such that $k(m)<m,(m-k(m)) \rightarrow \infty$, and $k(m) \sim m$ as $m \rightarrow \infty$. Then by Lemma 1 the finite sequence

$$
h_{m}=\left(H_{n_{m}}^{m-k(m)}, H_{n_{m}+1}^{m-k(m)}, \ldots, H_{n_{m}+k(m)}^{m-k(m)}\right)
$$

with length $\left|h_{m}\right|=k(m)+1$ is $(\mu, \varepsilon)$-binary with $\varepsilon=\mu_{m-k(m)}-\mu$. Since $\varepsilon \rightarrow 0$ and $\left|h_{m}\right| \rightarrow \infty$ as $m \rightarrow \infty$, item (a) of the theorem is proved.

(b) As in Eq. (25), with every $n_{m}^{(i)} \in P_{m}$ (see Eq. (11)) we associate a sequence $h_{m}^{(i)}$. Since $d_{m}<m$ and $\left(m-d_{m}\right) \rightarrow \infty$, one can assign in Eq. (25) $k(m)=d_{m}$ what means that

$$
\bigcup_{i=1}^{\infty} h_{m}^{(i)}=H_{m-d_{m}} .
$$

Since every $i$ th $h_{m}^{(i)}$ is $(\mu, \varepsilon)$-binary with $\varepsilon=\mu_{m-d_{m}}-\mu$, their union $H_{m-d_{m}}$ is also $(\mu, \varepsilon)$ binary with the same $\varepsilon$. Since $\varepsilon \rightarrow 0$ as $m \rightarrow \infty$, item (b) of the theorem is proved.

(c) The proof can be deduced from the previous item (b) and the example from Eq. (15). We present a more detailed proof. If Eq. (9a) holds, the proof of the theorem follows $a b$ absurdo, if one takes into account Eq. (2) and the periodicity of every $H_{m}$. Let us consider the case when Eq. (9b) holds. Since every $H_{m}$ is $T$-periodic, to prove that $H_{m}$ is $(\mu, \varepsilon)$ binary it suffices to prove that for some $n$ the finite sequence

$$
H_{n}^{m}, H_{n+1}^{m}, \ldots, H_{n+T}^{m}
$$

is $(\mu, \varepsilon)$-binary. This statement follows from Lemma 1 if one considers the sequence $H_{m+T}$ and chooses some $n \geq 1$ such that $H_{n}^{m+T} \geq \mu$ (cf. Eq. (18)): indeed, then Lemma 1 yields the result that the finite sequence (26) is $(\mu, \varepsilon)$-binary with $\varepsilon=\mu_{m-T}-\mu$. Then due to the $T$-periodicity of $H_{m}$ we see that $H_{m}$ is also $(\mu, \varepsilon)$-binary. Item (c) of the theorem is proved. Theorem 1 is proved.

Proof of Corollary 1 To prove the first point of Corollary 1, we note that since $X$ is periodic and the $f_{i}$ are rational, by considering the common denominator for $f_{1}, \ldots, f_{T}$ one can suppose that $X$ is a (periodic) sequence of natural numbers; then it follows that Eq. (9a) 
for such $X$ holds, and hence $\left(H_{m}\right)_{m \geq 1}$ is $\mu$-binary. The second point of Corollary 1 follows from Eq. (6). Indeed, due to the assumption on $\mathrm{H}$-independence none of the $H_{n}^{m}$ can take the value 0 , and hence by Theorem 1 (c) $\left(H_{m}\right)_{m \geq 1}$ cannot be $\mu$-binary and then Eq. (9a) is impossible; then the alternative option Eq. (9b) mentioned in Theorem 1(c) asserts that $\left(H_{m}\right)_{m \geq 1}$ is asymptotically $\mu$-binary. Corollary 1 is proved.

Competing interests

The authors declare that they have no competing interests.

\section{Authors' contributions}

AYS participated in the most part of results formulations, their proofs, and the manuscript preparation. RPA participated in the preparation of proofs of results and drafted the manuscript. RBB participated in preparing of the proofs, discussing signal processing, and drafting the manuscript. All authors read and approved the final manuscript.

\section{Author details}

'Institute for Informatics and Automation Problems, National Academy of Sciences of Armenia, Paruir Sevak St. 1, Yerevan, 0014, Armenia. ${ }^{2}$ Department of Mathematics, Texas A\&M University, Kingsville 700 University Blvd., Kingsville, TX 78363-8202, USA. ${ }^{3}$ Vision and Natural Computation Group, Vision Institute, University Pierre et Marie Curie-Paris 6, 17 rue Moreau, Paris, 75012, France.

\section{Acknowledgements}

AYS would like to thank Prof. H Niederreiter for his interest to this work and discussions. The authors thank the reviewers of the manuscript for their comments.

Received: 27 November 2013 Accepted: 23 January 2014 Published: 07 Feb 2014

\section{References}

1. Shahverdian, AY, Apkarian, AV: On irregular behavior of neuron spike trains. Fractals 7, 93-103 (1999)

2. Shahverdian, AY: The finite-difference method for analyzing one-dimensional nonlinear systems. Fractals 8, 49-65 (2000)

3. Shahverdian, AY, Apkarian, AV: A difference characteristic for one-dimensional nonlinear systems. Commun. Nonlinear Sci. \& Comput. Simul. 12, 233-242 (2007)

4. Shahverdian, AY: Minimal Lie algebra, fine limits, and dynamical systems. Rep. Armenian Natl. Acad. Sci. 112, 160-169 (2012)

5. Shahverdian, AY, Kilicman, A, Benosman, RB: Higher difference structure of some discrete processes. Adv. Differ. Equ. 2012, $202(2012)$

6. Miller, KD: In: Domany, E, van Hemmen, JL, Schulten, K (eds.) Models of Neural Networks, vol. 3. Springer, New York (1996)

7. Schuster, HG: Deterministic Chaos. Physik-Verlag, Weinheim (1984)

8. Dai, L, Wang, G: Implementation of periodicity ratio in analyzing nonlinear dynamic systems: a comparison with Lyapunov exponent. J. Comput. Nonlinear Dyn. 3, 1006-1015 (2008)

9. Dai, L: Nonlinear Dynamics of Piecewise Constant Systems and Implementation of Piecewise Constant Arguments. World Scientific, Singapore (2008)

10. Wu, GC, Baleanu, D: Discrete fractional logistic map and its chaos. Nonlinear Dyn. 75, 283-287 (2014)

11. Wu, GC, Baleanu, D, Zeng, SD: Discrete chaos of fractional sine and standard maps. Phys. Lett. A 378, 484-487 (2014)

12. Tarasov, VE, Edelman, M: Fractional dissipative standard map. Chaos 20, 023127 (2010)

13. Hardy, GH: Divergent Series. Clarendon, Oxford (1949)

14. Phelps, RP: Lectures on Choquet Theorems. Van Nostrand, Princeton (1966)

15. Dynkin, EB, Yushkevich, AA: Theorems and Problems on Markov Processes. Nauka, Moscow (1967)

16. Gamelin, TW: Uniform Algebras. Prentice Hall, Englewood Cliffs (1969)

17. Mergelyan, SN: Some Results in the Theory of Uniform and Best Approximation by Polynomials and Rational Functions. Appendix to the Russian Translation of the Walsh, JL: Interpolation and Approximation by Rational Functions in the Complex Domains. Amer. Math. Soc., Providence (1960), Moscow (1961) (in Russian)

10.1186/1687-1847-2014-60

Cite this article as: Shahverdian et al.: The bistability of higher-order differences of discrete periodic signals. Advances in Difference Equations 2014, 2014:60 